\title{
Epothilone B Facilitates Peripheral Nerve Regeneration by Promoting Autophagy and Migration in Schwann Cells
}

\section{OPEN ACCESS}

Edited by:

Kirsten Haastert-Talini, Hannover Medical School, Germany

Reviewed by: Arthur W. English, Emory University, United States Stefania Raimondo, University of Turin, Italy

${ }^{*}$ Correspondence: Hao Zhang zhanghaodoctor@hotmail.com Zhuojing Luo zhuojing@@163.com Jinghui Huang huangjh@fmmu.edu.cn

tThese authors have contributed equally to this work

Specialty section:

This article was submitted to Non-Neuronal Cells, a section of the journal Frontiers in Cellular Neuroscience

Received: 12 January 2020 Accepted: 28 April 2020 Published: 26 May 2020

\section{Citation:}

Zhou J, Li S, Gao J, Hu Y, Chen S, Luo $X$, Zhang H, Luo Z and Huang J (2020) Epothilone B Facilitates

Peripheral Nerve Regeneration by Promoting Autophagy and Migration in Schwann Cells. Front. Cell. Neurosci. 14:143. doi: 10.3389/fncel.2020.00143

\begin{abstract}
Jianhua Zhou ${ }^{1 \dagger}$, Shengyou $\mathrm{Li}^{2 \dagger}$, Jianbo Gao ${ }^{2 \dagger}$, Yawei Hu${ }^{1}$, Shaochu Chen ${ }^{1}$, Xinle Luo ${ }^{1}$, Hao Zhang ${ }^{1 *}$, Zhuojing Luo ${ }^{2 *}$ and Jinghui Huang ${ }^{2 *}$

${ }^{1}$ Department of Spine Surgery, The People's Hospital of Longhua District of Shenzhen, Shenzhen, China, '²Department of Orthopedics, Xijing Hospital, Fourth Military Medical University, Xi'an, China
\end{abstract}

The search for drugs that can facilitate axonal regeneration and elongation following peripheral nerve injury has been an area of increasing interest in recent years. Epothilone $\mathrm{B}$ (EpoB) is an FDA-approved antineoplastic agent, which shows the capacity to induce $\alpha$-tubulin polymerization and to improve the stability of microtubules. Recently, it has been increasingly recognized that $\mathrm{EpoB}$ has a regenerative effect in the central nervous system. However, the information currently available regarding the potential therapeutic effect of EpoB on peripheral nerve regeneration is limited. Here, we used a rat sciatic crush injury model system to determine that EpoB strikingly improved axonal regeneration and recovery of function. Also, EpoB (1 nM) did not result in significant apoptosis in Schwann cells (SCs) and showed little effect on their viability either. Interestingly, EpoB (1 nM) significantly enhanced migration in SCs, which was inhibited by autophagy inhibitors 3-methyladenine (3-MA). Since PI3K/AKt signaling has been implicated in regulating autophagy, we further examined the involvement of PI3K/Akt in the process of EpoB-induced SC migration. We found that EpoB (1 nM) significantly inhibited phosphorylation of PI3K and Akt in SCs. Further studies showed that both EpoB-enhanced migration and autophagy were increased/inhibited by inhibition/activation of PI3K/Akt signaling with LY294002 or IGF-1. In conclusion, EpoB can promote axonal regeneration following peripheral nerve injury by enhancing the migration of SCs, with this activity being controlled by PI3K/AKt signaling-mediated autophagy in SCs. This underscores the potential therapeutic value of EpoB in enhancing regeneration and functional recovery in cases of peripheral nerve injury.

Keywords: epothilone B, autophagy, migration, peripheral nerve injury, remyelination

\section{INTRODUCTION}

Peripheral nerve injuries (PNIs) occur relatively frequently as a result of inflammatory, infectious, traumatic, or iatrogenic causes, resulting in chronic morbidity. PNIs are thought to affect millions of individuals globally each year, resulting in substantial social and economic burdens and rates of disability (Yu et al., 2015). PNI can lead to sensory or motor dysfunction as well as to tissue atrophy that cannot be reversed (Chen et al., 2019). While there are biological mechanisms capable 
of facilitating spontaneous recovery within the adult mammalian peripheral nervous system, the process is complex, and often results in unsatisfactory outcomes (Chen et al., 2015). The current gold standard treatments for PNIs are thus combinations of surgical repair, nerve bridging, and nerve grafting (Meyer et al., 2016). However, surgical procedures only restore the pathway through which regenerated axons reach their target organs (Huang et al., 2012; Chen et al., 2015) and do not improve the regenerative capability of the injured neurons. It is thus vital to explore drugs capable of promoting nerve regeneration to allow restoration of function after PNI.

Epothilone B (EpoB) is an antineoplastic agent with FDA approval, which shows the capacity to improve the stability of microtubules and to promote $\alpha$-tubulin polymerization (Ballatore et al., 2012). EpoB can promote OV-90 and ABT-737 ovarian cancer cells apoptotic death via respective signaling through the Apo-2L/TRAIL and PI3K/Akt/mTOR pathways (Rogalska and Marczak, 2015; Li et al., 2016). More recently, EpoB has exhibited a regenerative effect in the central nervous system. Systemic administration of subtoxic EpoB doses enhances axonal regeneration and attenuates fibrotic scarring following an injury to the spinal cord by abrogating meningeal fibroblast polarization and migration (Ruschel et al., 2015). Intraperitoneal low-dose EpoB administration has been shown to suppress axonal microtubule deterioration and improve cognitive function in a murine tauopathy model (Ballatore et al., 2012). Furthermore, systemic EpoD administration has been suggested to protect against a murine MPTP-induced parkinsonism model (Cartelli et al., 2013). A recent study found that $\mathrm{EpoB}$ alleviates nigrostriatal pathway damage while enhancing motor functionality following intracerebral hemorrhage in mice (Yang et al., 2018). Taken together, these results confirm that $\mathrm{EpoB}$ has a regenerative effect in the central nervous system. However, reports of the effect of EpoB on nerve regeneration after nerve injury in the peripheral nervous system have been lacking.

Herein, we conducted a systematic assessment of how EpoB affects axonal regeneration and remyelination in a rat model of PNI. We explored the effect of EpoB on Schwann cells (SCs) and further revealed the underlying mechanisms. Our results show that the administration of EpoB improves axonal regeneration and functional recovery in a rat model of sciatic crush injury. We further show that EpoB (1 nM) significantly enhances migration in SCs, which is controlled via PI3K/Akt signaling-mediated autophagy. This work highlights the potential therapeutic value of EpoB in enhancing regeneration and functional recovery in PNI.

\section{MATERIALS AND METHODS}

\section{PNI Modeling}

Adult male Sprague-Dawley (SD) rats (200-250 g) were obtained from the Laboratory Animal Center of the Fourth Military Medical University. Animals were housed following the NIH Guide for the Care and Use of Laboratory Animals, with the Animal Experimentation Ethics Committee of The Fourth Military Medical University having approved all studies described herein. Briefly, five rats were housed in each cage in a climate-controlled facility $\left(23 \pm 2{ }^{\circ} \mathrm{C} ; 35 \%-60 \%\right.$ humidity; $12 \mathrm{~h}$ light/dark cycle).

The rat sciatic nerve injury model was generated as described in prior studies, with small modifications (Li R. et al., 2017). Briefly, $40 \mathrm{mg} / \mathrm{kg}$ of $1 \%$ pentobarbital sodium was i.p. injected into animals to achieve anesthesia, after which the left sciatic nerve was exposed through the generation of a $1 \mathrm{~cm}$ cut in the mid-thigh. Two vascular clips (30 g for $50 \mathrm{~s}$; Oscar, Guangzhou, China) were used to compress the sciatic nerve, thereby inducing a moderate crushing injury. One of these clips was located $2 \mathrm{~mm}$ distal to the lesion, with the other being $7 \mathrm{~mm}$ proximal to the trifurcation of the sciatic nerve. The wound site was next closed using nondegradable sutures. Animals were then randomized into two model treatment groups: (1) control; and (2) EpoB ( $n=10$ per group). Sham controls underwent identical surgery but were not subjected to a crush injury. Animals in the EpoB group were administered $1 \mathrm{ml}$ of $150 \mu \mathrm{g} / \mathrm{ml} \mathrm{EpoB}$ via intraperitoneal injection for 7 days (once/day) following injury. Identical volumes of saline were administered to all other animals.

\section{Walking Track Assessment}

At 1,2, 3, and 4 weeks post-injury, the behavior of rats was evaluated via walking track assessment. Briefly, animals were allowed to walk down a wooden alley $\left(5.0 \times 8.0 \times 45.0 \mathrm{~cm}^{3}\right)$ that had a dark goal box positioned at the far end. To monitor walking behavior, the plantar surfaces of these rat's feet were coated with acrylic paint, and white paper was used to cover the surface of the wooden alley. This enabled the visualization of anatomical landmarks within the resultant footprints. The prints were analyzed and utilized for the calculation of the sciatic functional index (SFI). This was repeated three more times to ensure footprints were clear enough for utilization.

\section{Von Frey Hair Test}

Von Frey hair test was performed for all animals at 1, 2, 3, and 4 weeks post-surgery. Before testing animals were given $1 \mathrm{~h}$ to habituate to a glass cubicle, with the bottom of this container containing wire mesh and a surface filled with $5 \times 5 \mathrm{~mm}^{2}$ holes that were $1 \mathrm{~mm}$ apart from one another. Rat tactile thresholds were evaluated by placing Von Frey filaments (NC12775; North Coast Medical, San Francisco, CA, USA) perpendicular to the planta pedis and pressing until a perceptible $\sim 90^{\circ}$ bend was evident for 6-8 s. At the threshold, the animal is expected to respond by quickly flicking its paw away from the hair. Forces sufficient to induce dodging reactions, such as the withdrawal of the paw, licking or avoidance behaviors, jittering, or the like were recorded as a means of calculating withdrawal threshold values. This was repeated thrice by the same investigator, with an interval of $15 \mathrm{~min}$.

\section{Histological Analysis}

Isolated sciatic nerve portions containing the injured site were separated into two sections at 28 days after sciatic crush injury. One section $(\sim 1 \mathrm{~cm}$; Figure 1A) was fixed overnight using cold $4 \%$ paraformaldehyde in PBS, followed by paraffin embedding and cutting into longitudinal sections for 


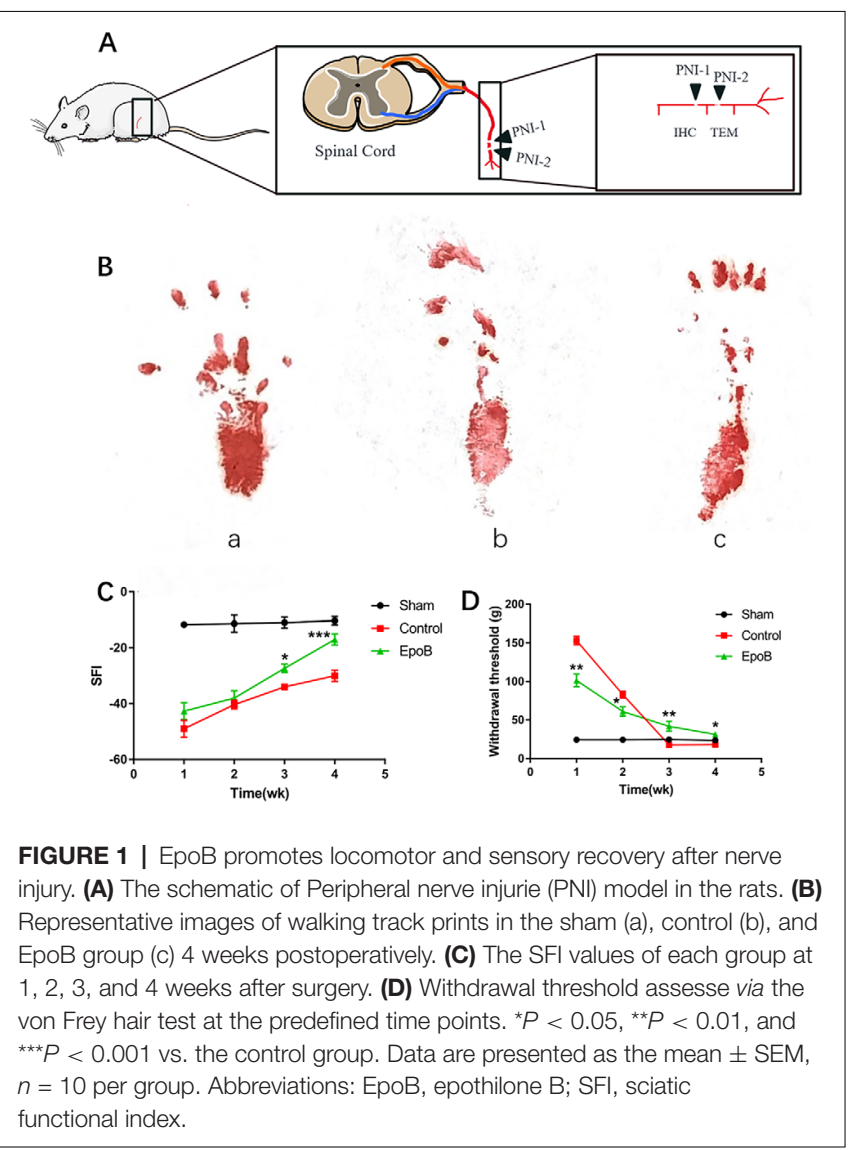

immunofluorescence staining. The sections were incubated with the primary antibodies at $4^{\circ} \mathrm{C}$ overnight. Primary antibodies were specific for NF-200 and MBP (Abcam, Cambridge, UK). Next, secondary antibodies were added for $1 \mathrm{~h}$ at room temperature (Cwbio; 1:10,000). Six sections per nerve group were analyzed as a means of assessing axonal regeneration via confocal fluorescent microscopy (A1+, Nikon, Japan) and at least six images in each nerve section were analyzed by the ImageJ software.

Another $2 \mathrm{~mm}$ segment (Figure 1A) was submerged in 3\% glutaraldehyde, after which it underwent additional fixation with a solution of $1 \%$ osmium tetroxide in $0.1 \mathrm{M}$ sodium cacodylate buffer ( $\mathrm{pH}$ 7.3). Segments were then dehydrated, epoxy resinembedded, and the distal nerve was cut to prepare transverse ultrathin sections $(50 \mathrm{~nm})$. Uranyl acetate and lead citrate were then used to stain these sections, followed by TEM (TECNAI Spirit, FEI, USA) evaluation. A total of six sections per nerve group were assessed at random to evaluate axonal regeneration, with six random fields of view per sample being analyzed. We measured myelin thickness (MT) and axonal diameter (AD) using the ImageJ software. Fiber diameter (FD) was calculated according to the formula: $\mathrm{FD}=2 \times \mathrm{MT}+\mathrm{AD}$. The G-ratio $(\mathrm{AD} / \mathrm{FD})$ was also determined as a structural indicator including axonal myelination.

\section{Cell Culture and Immunofluorescence}

Primary SCs were collected from newborn SD rats provided by the Laboratory Animal Center of the Fourth Military Medical
University, according to previously published procedures (Xia et al., 2016). Briefly, animals were euthanized within 3 days of birth using cold alcohol (75\%) followed by sciatic nerve extraction. The epineurium of the sciatic nerves was stripped and the endoneurium was combed under a microscope (Olympus, Tokyo, Japan). Scalpels were then used to mince these nerves into small pieces, after which $0.25 \%$ trypsin and $0.03 \%$ collagenase (Sigma-Aldrich, St. Louis, MO, USA) were used to digest them for $30 \mathrm{~min}$ at $37^{\circ} \mathrm{C}$. Isolated cells were then cultured in DMEM (Thermo Fisher Scientific, Waltham, MA, USA) supplemented with $15 \%$ FCS (Thermo Fisher Scientific) and penicillin/streptomycin. At $24 \mathrm{~h}$ post-plating, cells were then treated for $48 \mathrm{~h}$ using cytosine arabinoside $\left(10^{-5} \mathrm{M}\right)$ as a means of preventing the proliferation of fibroblasts. Cells were then transferred to DMEM containing $2 \mathrm{mM}$ forskolin (Sigma-Aldrich), $20 \mu \mathrm{g} / \mathrm{ml}$ bovine pituitary extract (Biomedical Technologies, MA, USA), and 15\% FCS.

Cells were put into 6-well plates with coverslips for $24 \mathrm{~h}$, followed by $4 \%$ PFA fixation and $0.15 \%$ Triton-mediated permeabilization. Then they were washed with PBS thrice and incubated using primary antibodies $(1: 100)$ overnight at $4^{\circ} \mathrm{C}$. These antibodies were specific for S-100 and p75 (Abcam, Cambridge, UK). Next, secondary antibodies (Cwbio; 1:10,000) were added for $1 \mathrm{~h}$, followed by additional washing using PBS and 4,6-diamidino-2-phenylindole (DAPI; Sigma-Aldrich) staining of cell nuclei. The ImageJ software was then used for enumerating p75NTR+ and S-100+ cells, revealing cell preparations to be highly pure ( $>96 \% \mathrm{SCs}$ ) as determined based upon the assessment of 5 random fields of view via confocal microscope (A1+, Nikon, Japan).

\section{Cell Viability Assay}

SCs were added to 96-well plates (3,000/well, in quadruplicate). After $24 \mathrm{~h}$, EpoB was added at appropriate concentrations for $24 \mathrm{~h}$, after which each well was treated using 10- $\mu \mathrm{l}$ of Cell Counting Kit 8 (CCK8; Dojindo, Kumamoto, Japan) for $1 \mathrm{~h}$ at $37^{\circ} \mathrm{C}$. Absorbance at $450 \mathrm{~nm}$ was then evaluated via a microplate reader.

\section{Apoptosis Assay}

SCs were plated in 6-well plates $\left(8 \times 10^{4} /\right.$ well $)$ for $24 \mathrm{~h}$, after which fresh media with or without $1 \mathrm{nM}$ EpoB was added for an additional $24 \mathrm{~h}$. In some experiments, 3-methyladenine (3-MA; $0.5 \mathrm{mM})$, IGF-1 (100 ng/ml), or LY294002 $(20 \mu \mathrm{M}$; MedChemExpress, Shanghai, China) was added to the cultures and then maintained in the same media for the entire experiment. Cells were then suspended in $195 \mu$ l Annexin V-FITC binding buffer to which $5 \mu \mathrm{l}$ Annexin V-FITC and $10 \mu \mathrm{l}$ PI (Beyotime, Shanghai, China) was added. Following a $20 \mathrm{~min}$ staining period, flow cytometry (Beckman Instruments, Pasadena, CA, USA) was used to analyze samples in triplicate.

\section{Cell Cycle Assay}

PI (Beyotime) was used to evaluate cell cycle progression. Briefly, isolated SCs were fixed with $70 \%$ ethanol for $2 \mathrm{~h}$ at $4^{\circ} \mathrm{C}$, after which PI was added before flow cytometric analysis (Beckman Instruments, Pasadena, CA, USA). The samples were assessed in triplicate. 


\section{Migration Analysis}

Transwell chambers (Corning, New York, NY, USA) with an $8 \mu \mathrm{m}$ pore size were used to evaluate the impact of EpoB on SC migration. Briefly, SCs were added to the upper chamber $\left(6 \times 10^{4} /\right.$ well), while $500 \mu \mathrm{l}$ of complete media supplemented with appropriate drugs was placed in the lower chamber. Following a $10 \mathrm{~h}$ incubation, a cotton swab was used to remove cells from the upper chamber surface, while remaining cells were fixed in 4\% PFA and stained for 15 min using crystal violet. A total of five random fields of view per chamber were then assessed via microscopy (Olympus, Tokyo, Japan).

\section{Western Blotting}

RIPA buffer (Beyotime) containing phosphatase and protease inhibitors was used to lyse cells for $5 \mathrm{~min}$. The resultant lysates were spun for $15 \mathrm{~min}$ at $12,000 \mathrm{~g}$, and a BCA Assay Kit (Beyotime) was used to quantify protein levels. Samples were then boiled for $15 \mathrm{~min}$ and separated via SDS-PAGE before transfer onto PVDF membranes (Thermo Fisher Scientific). Blots were then blocked using 5\% non-fat milk in TBST, probed overnight with anti-p-PI3K (Cell Signaling Technology, Boston, MA, USA), anti-LC3B, anti-p62, anti-PI3K, anti-Akt, anti-p-Akt (Abcam, Cambridge, UK) and anti- $\beta$-actin (Cwbio, China) at $4^{\circ} \mathrm{C}$, washed thrice in TBST, probed with secondary HRP-linked antibodies for $1 \mathrm{~h}$, and assessed via chemiluminescent analysis (Bio-Rad). Samples were analyzed in triplicate.

\section{Transmission Electron Microscopy}

A TEM (TECNAI Spirit, FEI, USA) was used to determine the numbers of autophagic vesicles in SCs. In brief, cells were put in fixative for transmission electron microscopy at $4^{\circ} \mathrm{C}$ for $2 \mathrm{~h}$, washed thrice in PBS, and post-fixed for $2 \mathrm{~h}$ using $1 \% \mathrm{OsO}_{4}$ in $0.1 \mathrm{M}$ PBS ( $\mathrm{pH}$ 7.4). Then $\mathrm{OsO}_{4}$ was removed from the sample, and it was dehydrated with an ethanol gradient with epoxy resin embedding. We conducted polymerization for $48 \mathrm{~h}$ at $60^{\circ} \mathrm{C}$, followed by cutting into $70 \mathrm{~nm}$ ultrathin sections. Post-staining was next conducted for 15 min using uranyl acetate in pure ethanol, after which lead citrate was used and TEM (TECNAI Spirit, FEI, USA) was used for analysis. Data were quantified and analyzed by Image J software (50 cells were counted in a blind manner). At least three individual experiments were performed.

\section{Statistical Analysis}

Data are means \pm standard deviations (SDs) and were compared via one-way ANOVAs using SPSS 13.0 (IBM, IL, USA). Tukey's post hoc test and Dunnett's test were used to compare significant results (GraphPad Prism 7.0; GraphPad Software, CA, USA). $P<0.05$ was the significance threshold.

\section{RESULTS}

\section{EpoB Promotes Functional Recovery Following PNI}

To evaluate the impact of EpoB on peripheral nerve regeneration, rats with sciatic crush injury were intraperitoneally injected with $150 \mu \mathrm{g}$ EpoB daily for 1 week. Locomotor recovery throughout the 4 weeks was evaluated by walking track analysis, which showed that the SFI progressively improved over time (Figure 1B). SFI did not differ significantly between the control and EpoB groups within the first 2 weeks. At $3(-27.50 \pm 1.58)$ and $4(-17.32 \pm 2.17)$ weeks after injury and treatment, the SFI in the EpoB group was significantly elevated relative to the control group $(P<0.05,-33.94 \pm 1.06 ; 3$ weeks post-injury; $P<0.05,-29.73 \pm 1.97 ; 4$ weeks post-injury), respectively (Figure 1C), suggesting superior motor functional recovery was achieved by the EpoB treatment.

Recovery of the sensory function of the injured hindlimb was evaluated throughout the 4 weeks using the Von Frey hair test. The results revealed that the withdrawal threshold in the EpoB group gradually decreased over 4 weeks, indicating that the recovery of sensory function was achieved by EpoB treatment after nerve crush injury. Also, the EpoB-treated rats showed a lower withdrawal threshold than the control rats within the first 2 weeks after injury (Figure 1D). The withdrawal threshold of the control group At $3(17.61 \pm 0.88)$ and $4(17.92 \pm 1.49)$ weeks after injury dramatically decreased to an even lower level than that of the sham-injury group $(P<0.05,17.61 \pm 0.88$; 3 weeks after injury; $P<0.05,17.92 \pm 1.49 ; 4$ weeks after injury), respectively (Figure 1D), indicating that hyperalgesia might be present after crush injury in the control group. Further investigations showed that the withdrawal threshold in the EpoB-treated group was significantly higher $(P<0.05$, $43.21 \pm 3.42$; 3 weeks after injury; $P<0.05,31.58 \pm 2.81 ; 4$ weeks after injury) than that in the control group at $3(17.78 \pm 1.64)$ and $4(18.01 \pm 1.33)$ weeks after injury (Figure 1D), indicating that EpoB is capable of ameliorating hyperalgesia at the later stage of nerve recovery. These findings suggest that EpoB treatment is capable of improving the recovery of sensory function after nerve crush injury.

\section{EpoB Enhances Axon Regeneration and Remyelination After PNI}

We next examined the impact of EpoB on nerve regeneration, which is the structural foundation for functional recovery (Frendo et al., 2019; Liu et al., 2019). To evaluate the impact of EpoB on neuronal regeneration, we co-stained for NF-200 (a neurofilament heavy subunit found in both large and small axons) and myelin basic protein (MBP; which is a marker of myelination) was performed on longitudinal sections (Figures 2A-L). The ratio of the positive area of NF-200 $(57.50 \% \pm 3.35)$ and total positive of $\mathrm{MBP}(66.34 \% \pm 2.74)$ in EpoB treated group was in the low range to that in the sham-treated group $(73.81 \% \pm 5.71$; NF-200 and $77.39 \% \pm 4.61$; MBP), which was significantly higher than that in the PNI control group $(29.51 \% \pm 3.31$; NF-200 and $29.82 \% \pm 1.58$; MBP; Figures $\mathbf{2 M}, \mathbf{N}$ ). These data indicated that the number of myelinated nerve fibers was significantly increased by EpoB compared to that in the PNI control group. All of these results suggest that EpoB administration contributes to the regeneration of myelinated nerve fibers after nerve crush injury.

The effect of EpoB on the myelination of regenerated axons was further evaluated by TEM (Figures 3A-F). A decreased thickness of the myelin sheath was observed in the PNI control group $(P<0.05$, Figure $3 \mathrm{G})$. In contrast, the thickness of the 

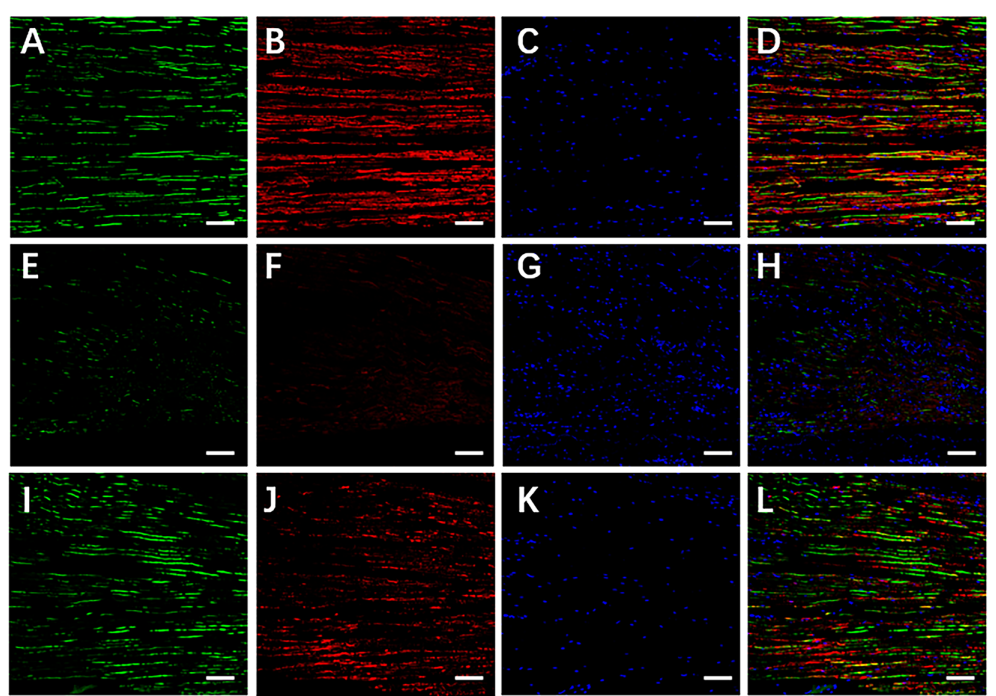
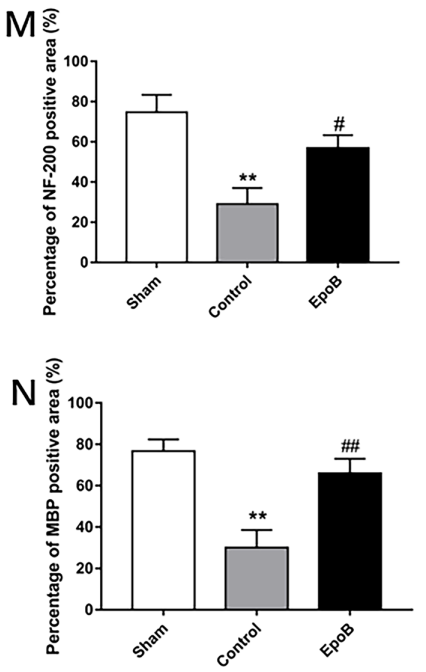

FIGURE 2 | EpoB promotes regeneration of myelinated nerve fibers. Representative regenerated axon images in the nerves stained for NF-200 (green), MBP (red), and DAPI (blue) in the sham (A-D), control (E-H) and EpoB (I-L) groups at 4 weeks after injury. Treatment of EpoB increased the ratio of positive area of NF-200 (M) and MBP (N). Scale bars: (A-L) $20 \mu \mathrm{m}$. ${ }^{\star \star} P<0.01$ vs. the sham group; ${ }^{\#} P<0.05,{ }^{\# \#} P<0.01$ vs. the control group. Abbreviations: EpoB, epothilone B; MBP, myelin basic protein; DAPI, 4,6-diamidino-2-phenylindole.

myelin sheath was significantly increased by EpoB treatment $(P<0.05$; Figure 3G). Further morphometric analysis showed that EpoB treatment led to a significantly greater MT and a lower G-ratio (axon/fiber ratio) after nerve crush injury $(P<0.05$; Figures $3 \mathbf{G}, \mathbf{H}$ ), indicated that $\mathrm{EpoB}$ is capable of improving remyelination of regenerated axons.

\section{The Effect of EpoB on the Biological Behaviors of SCs}

We additionally explored the potential impact of EpoB on SCs in isolated SC cultures, which showed a purity of $>96 \%$ (Figures 4A-D). EpoB (0.25-4.0 nM) had no significant effect on SC cell viability after $24 \mathrm{~h}$ of treatment $(P>0.05$; Figure $4 \mathrm{E})$. Therefore, EpoB at a moderate concentration ( $1 \mathrm{nM})$ was chosen for subsequent experiments. Further investigations showed that treatment with $1 \mathrm{nM}$ EpoB did not result in significant SC apoptosis (Figures 5A-C), and showed little effect on the SC cell cycle (Figures 5D-F), which were performed by flow cytometry assay. These findings show that EpoB has no significant effect on the apoptosis and cycle of SCs. To our surprise, we found that EpoB (1 nM) dramatically enhanced the number of migrated SCs to $82 \pm 4$ when the Control grope increase to $28 \pm 3$ in the transwell migration assay (Figures 5G-I). These results indicate that EpoB is capable of promoting the migration of SCs.

\section{EpoB Promotes SCs Migration via PI3K/Akt Signaling-Mediated Autophagy}

Autophagy has been implicated in cell migration. Herein, we examined the potential role of autophagy in EpoB-enhanced migration of SCs. We first examined whether EpoB could induce autophagy in SCs by western blot (Figure 6A). The results showed that EpoB significantly increased the level of LC3B II and reduced the level of P62 (two markers of autophagy) at $24 \mathrm{~h}$
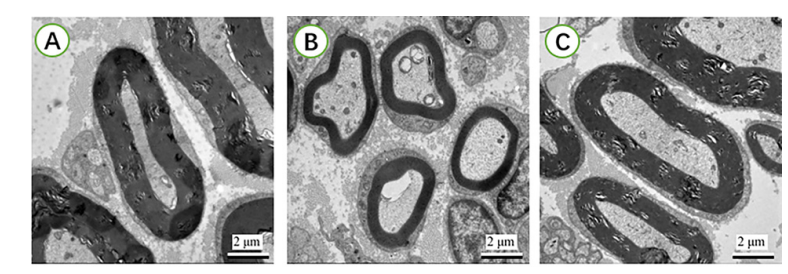

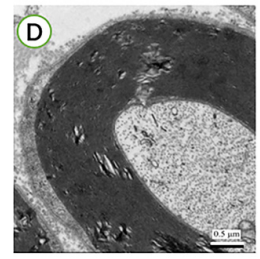

G

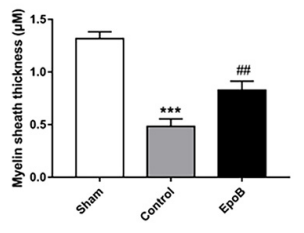

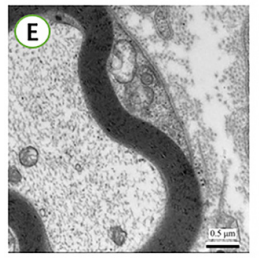

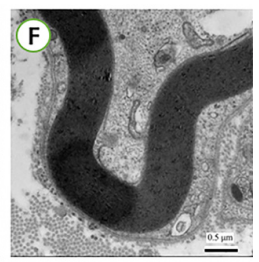

$\mathrm{H}$

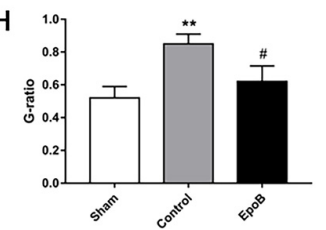

FIGURE 3 | EpoB promotes sciatic nerve remyelination after nerve injury. Representative TEM images of regenerated axons $(\mathbf{A}-\mathbf{C})$ and myelin sheaths (D-F) in the nerve segment of the sham $(\mathbf{A}, \mathbf{D})$, control $\mathbf{( B , E )}$ and EpoB $\mathbf{( C , F )}$ groups at 4 weeks after surgery, respectively. Quantification of the average myelin sheath thickness $(\mathbf{G})$ and the G-ratio $\mathbf{( H )}$. Scale bars: $(\mathbf{A}-\mathbf{C}) 2 \mu \mathrm{m}$; (D-F) $0.5 \mu \mathrm{m}$. ${ }^{\star \star} P<0.01$ and ${ }^{* \star} P<0.001$ vs. the sham group; ${ }^{\#} P<0.05$ and ${ }^{\# \# P}<0.01$ vs. the control group. Abbreviations: EpoB, epothilone B; TEM, transmission electron microscopy; G-ratio, axon/fiber ratio.

after treatment $(P<0.05$; Figure 6B). Also, transmission electron microscopy showed that EpoB markedly increased the number of autophagosomes $(5 \pm 1)$ in SCs after $24 \mathrm{~h}$ of the treatment 

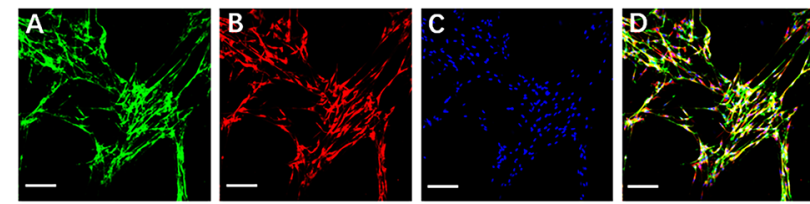

E

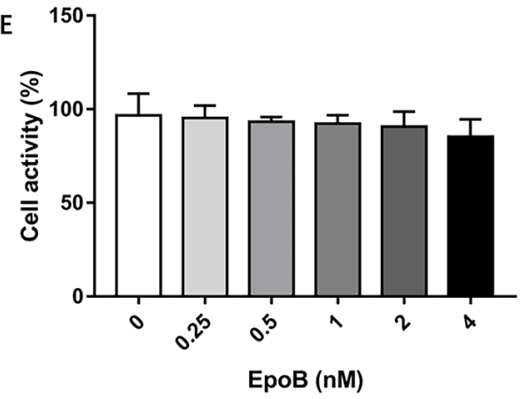

FIGURE 4 | Identification of SCs and measurement of their viability. Double-immunofluorescent staining of cultured SCs showing expression of p75 ${ }^{\text {NTR }}$ (green; A) and S-100 (red; B) with DAPI nuclear counterstaining (blue; C). (D) Merge images showed a SC purity $>96 \%$. (E) Effects of EpoB on SC viability assessed by the CCK-8 assay. Scale bar: (A-D) $100 \mu \mathrm{m}$.

Abbreviations: EpoB, epothilone B; CCK-8, Cell Counting Kit 8; SC, Schwann cell; DAPI, 4,6-diamidino-2-phenylindole.

compared to the control group ( $P<0.05,2 \pm 1$; Figures $6 \mathrm{C}-\mathbf{K})$. To further explore the role of autophagy in EpoB-enhanced migration of SCs, the effect of 3-MA (an autophagy inhibitor) on cell migration was examined (Figures 6C-J). It was found that 3-MA significantly inhibited EpoB-induced autophagosome production $(P<0.05$; Figure 6K), and abolished EpoB-enhanced migration in SCs $(P<0.05$; Figures 6L-P). Overall, these results suggest that autophagy was induced by EpoB and that this autophagy was involved in EpoB-enhanced migration in SCs.

The PI3K/Akt signaling pathway has been shown to regulate autophagy in previous studies (Zhao et al., 2017; Ho and Gorski, 2019). In the present study, we further explored the role of PI3K/Akt signaling in autophagy-mediated migration by EpoB in SCs. First, it was found that EpoB significantly reduced the $\mathrm{p}$-PI3K and $\mathrm{p}$-Akt expression in SCs $(P<0.05$; Figures 7A,B), suggesting that $\mathrm{EpoB}$ is capable of inhibiting the PI3K/Akt pathway. To further confirm the effect of PI3K/Akt signaling during EpoB-induced autophagy and migration in SCs, we evaluated the autophagy (Figures 7J-Q) and migration of SCs (Figures 7D-H) after treatment with a PI3K/Akt signaling enhancer (IGF-1; Wang L. et al., 2019) or inhibitor (LY294002; Li et al., 2019). It was found that IGF-1 significantly reduced the number of autophagosomes $(P<0.05$; Figure 7C) and inhibited migration of SCs $(P<0.05$; Figure $7 \mathbf{I})$ induced by EpoB. In contrast, LY294002 further increased cell autophagy in the presence of $\mathrm{EpoB}(P<0.05$; Figure $7 \mathrm{C})$. In addition, it was shown that LY294002 significantly increased migration in SCs; this effect was significantly reversed by the autophagy inhibitor 3-MA $(P<0.05$; Figure 7I). Those findings show that PI3K/Akt signaling plays an important role in EpoB-induced autophagy, which is involved in EpoB-enhanced migration in SCs.

\section{DISCUSSION}

EpoB has been widely accepted as an antineoplastic agent (Shin and Kwon, 2017; Cheng et al., 2018). Recent studies have increasingly recognized its regenerative effect in the central nervous system. Herein, the role of EpoB in nerve regeneration, and the mechanism underlying this, were investigated in the peripheral nervous system for the first time. The conclusions of the present study can be summarized as follows: (1) EpoB promotes nerve regeneration and remyelination, and of enhances functional recovery after nerve crush injury, suggesting that EpoB has a strong regenerative effect in the peripheral nervous system; (2) EpoB strongly promotes SC migration, a process which involves EpoB-enhanced autophagy; (3) EpoB inhibits the PI3K/Akt signaling pathway, which regulates the autophagymediated migration of SCs by EpoB. All of these findings indicate that $\mathrm{EpoB}$ plays a regenerative role after nerve injury in the peripheral nervous system, highlighting its therapeutic value in enhancing regeneration and functional recovery in cases of PNI.

PNI is highly prevalent in clinical settings, and generally results in nonreversible tissue atrophy and dysfunction, thereby extolling a significant burden on affected individuals, their families, and their societies (Johnson et al., 2005). Although recent research has made great advances in approaches to peripheral nerve repair, including nerve transplantation and the construction of nerve transplants (Fujimaki et al., 2020), the prognosis of patients with PNI remains poor. Moreover, surgical procedures that restore the anatomical continuity of the injured peripheral nerve have advanced, but show little effect on improving the regenerative capability of the injured neurons. Therefore, the search for a novel therapy that can enhance axonal regeneration has become urgent, in order to improve functional recovery after PNI.

EpoB belongs to the epothilone class of drugs, which are the group of microtubule-stabilizing agents. At present, EpoB has been approved for cancer therapy in clinics (Brogdon et al., 2014). Interestingly, many studies have identified the regenerative effect of $\mathrm{EpoB}$ in the central nervous system (Ballatore et al., 2012; Ruschel et al., 2015). In an animal model of spinal cord injury, it was found that systemic subtoxic EpoB dosing improves axonal regeneration while also attenuating fibrotic scarring (Ruschel et al., 2015). Similarly, in a mouse model of intracerebral hemorrhage, EpoB has been shown to improve the integrity of the nigrostriatal pathway neural circuit, increase the number of dopaminergic neurons, and enhance the recovery of fine motor function (Ruschel et al., 2015). Despite these findings relating to the central nervous system, the effect of EpoB on peripheral nerve regeneration has not been studied thus far. In the present study, we found that EpoB is capable of promoting nerve regeneration and remyelination, and of enhancing function recovery after nerve crush injury. This suggests that EpoB has a strong regenerative effect in the peripheral nervous system. However, the mechanisms underlying the beneficial effect of $\mathrm{EpoB}$ on nerve regeneration in the peripheral nervous system are unclear.

It was reported that Wallerian degeneration occurs after peripheral nerve injury. Axonal lesions undergo degeneration 

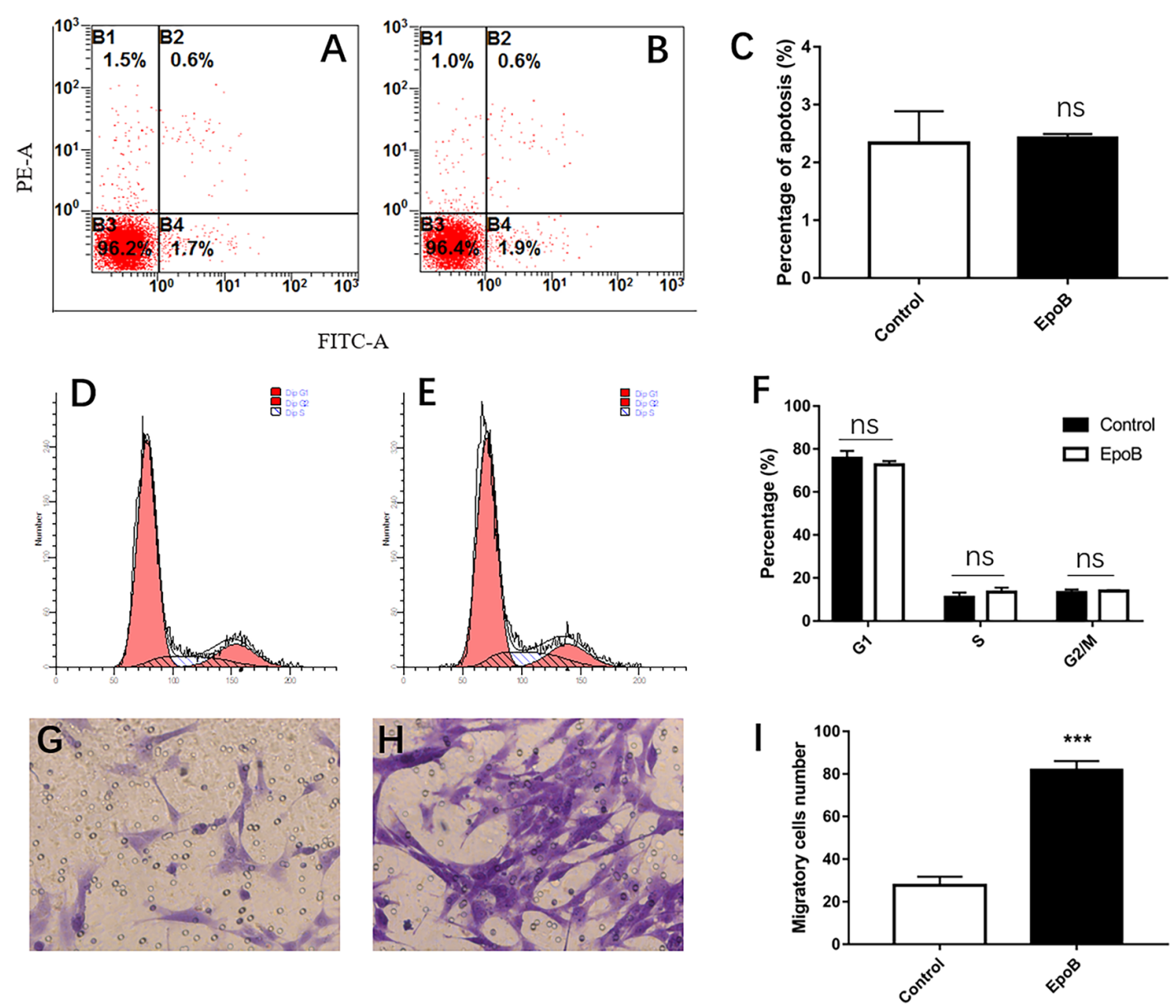

FIGURE 5 | Effects of EpoB on SCs. EpoB has no effect on SC apoptosis (A-C) or the SC cell cycle (D-F) assessed by flow cytometry. (G-I) EpoB significantly promotes migration of SCs by in the transwell assay. ${ }^{\star \star} P<0.001$ compared to the control group. Abbreviations: EpoB, epothilone B; SC, Schwann cell; ns, no significant.

and fragmentation, with myelin undergoing transformation into a neutral fat that can be taken up by macrophages. Axonal and myelin sheath debris also produce ovoids that are eventually broken down until no longer evident (Campbell, 2008). SCs are glial cells of the peripheral nervous system, which are capable of enhancing nerve regeneration in both the peripheral and central nervous system (Aszmann et al., 2008; Novikova et al., 2008). SCs play a key role in facilitating the regeneration of nervous system tissue given that they serve as a soruce of bioactive compounds that can support axonal migration and the release of compounds capable of controlling axonal outgrowth and remyelination (Zhang et al., 2009). In addition, the migratory ability of SCs has been widely accepted as a key process for guiding axonal elongation. In PNI, especially in peripheral nerve gap injury, axonal regeneration in vivo is limited by the extent of SC migration (Torigoe et al., 1996). Therefore, many studies have attempted to evaluate the feasibility of enhancing peripheral nerve regeneration by promoting SC migration. It has been shown that matrix metalloproteinase 7 (MMP-7) promotes axonal regeneration and remyelination by enhancing
SC migration after sciatic nerve injury in rats (Wang $H$. et al., 2019). In addition, enhancing SC migration using concentration gradients of laminin-derived peptides has been shown to support axonal regeneration over a large nerve gap, further confirming the role of SC migration in axonal regeneration (Motta et al., 2019). Herein, to our surprise, EpoB (1 nM) showed little effect on the viability or apoptosis of SCs, but significantly enhanced $\mathrm{SC}$ migration. This indicates that EpoB might promote axonal regeneration by enhancing $\mathrm{SC}$ migration.

Autophagy is a key pathway that controls the breakdown of proteins and organelles, and it plays key roles in maintaining approrpaite cellular homeostasis via controlling catabolic activities in response to stress to improve energy and amino acid availability (Mizushima et al., 2008). Autophagy is associated with Alzheimer's disease, Huntington's disease and Parkinson's disease (Bar-Yosef et al., 2019). In addition, autophagy is critical in the remodeling of presynaptic and postsynaptic constituents to sustain neuronal function (Gerónimo-Olvera and Massieu, 2019). An increasing number of studies have identified the role of autophagy in regulating cell migration, with diverse functions 
A

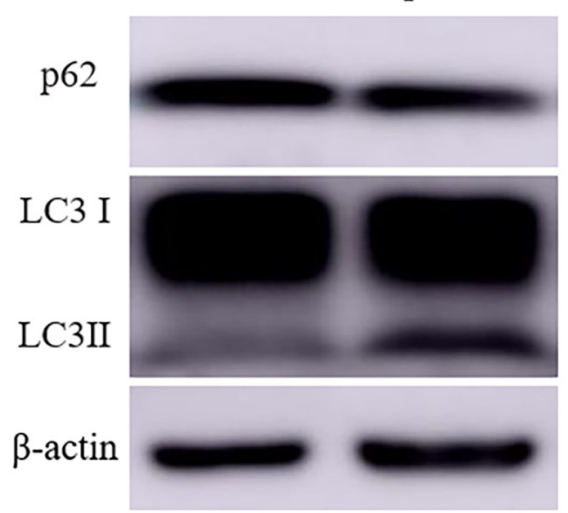

B

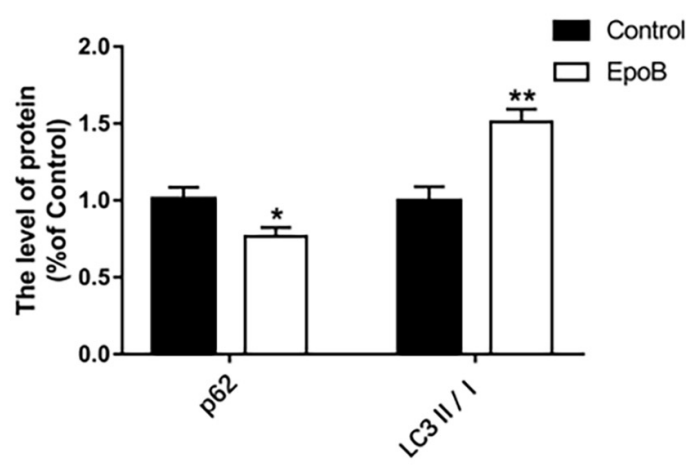

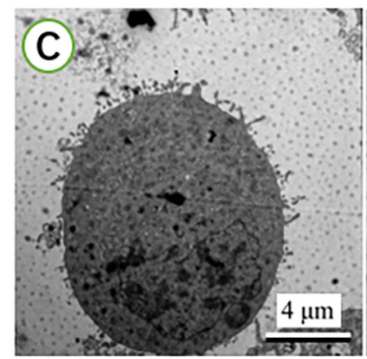
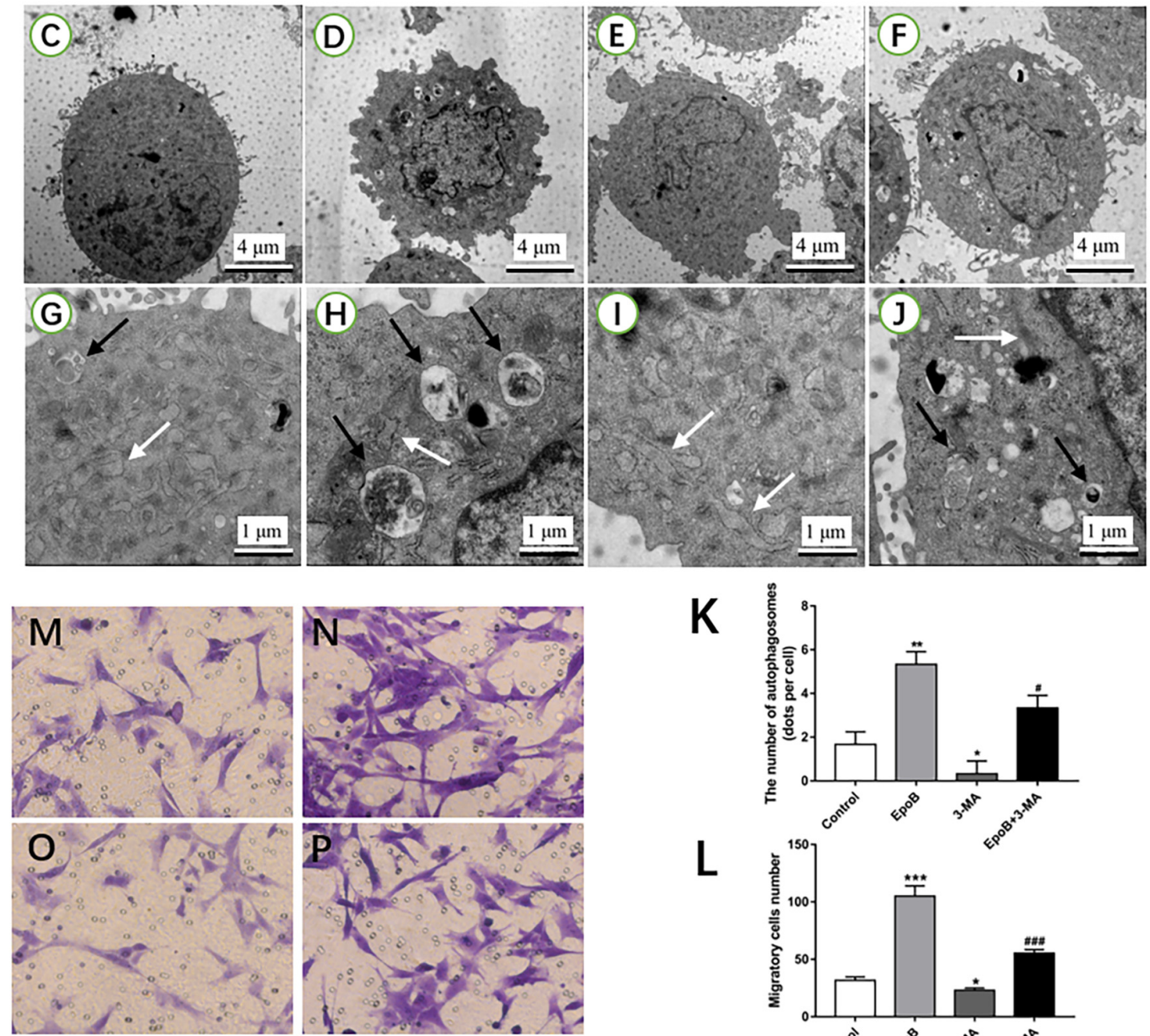

L

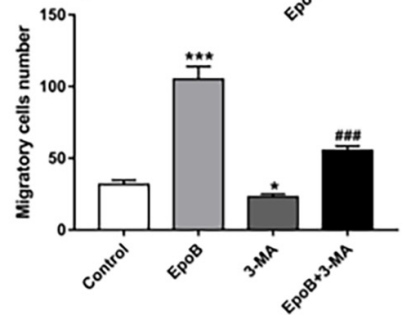

FIGURE 6 | EpoB increases migration of SCs by promoting autophagy. Representative western blotting (A) and data analysis of LC3 and p62 in each group (B). TEM analysis showing the presence of autophagosomes in SCs from the control (C, G), EpoB (D,H), 3-MA (E,I), and EpoB+3-MA (F,J) groups. White arrowheads indicate normal endoplasmic reticula; black arrowheads indicate double-membraned autophagosomes. (K) Quantification of the total number of autophagosomes in each group. Transwell analysis of migratory cells in the control (M), EpoB (N), 3-MA (O), and EpoB+3-MA (P) groups at $24 \mathrm{~h}$ after treatment. (L) Quantification of the total number of migratory cells in each group. Scale bars: (C-F) $4 \mu \mathrm{m}$; (G-J) $1 \mu \mathrm{m}$. ${ }^{\star} P<0.05,{ }^{\star \star} P<0.01$, and ${ }^{\star \star \star} P<0.001$ compared to the control group; ${ }^{\#} P<0.05$ and \#\#\# $P<0.001$ compared to the EpoB group. Abbreviations: EpoB, epothilone B; 3-MA, 3-methyladenine; SCs, Schwann cells; TEM, transmission electron microscopy. 

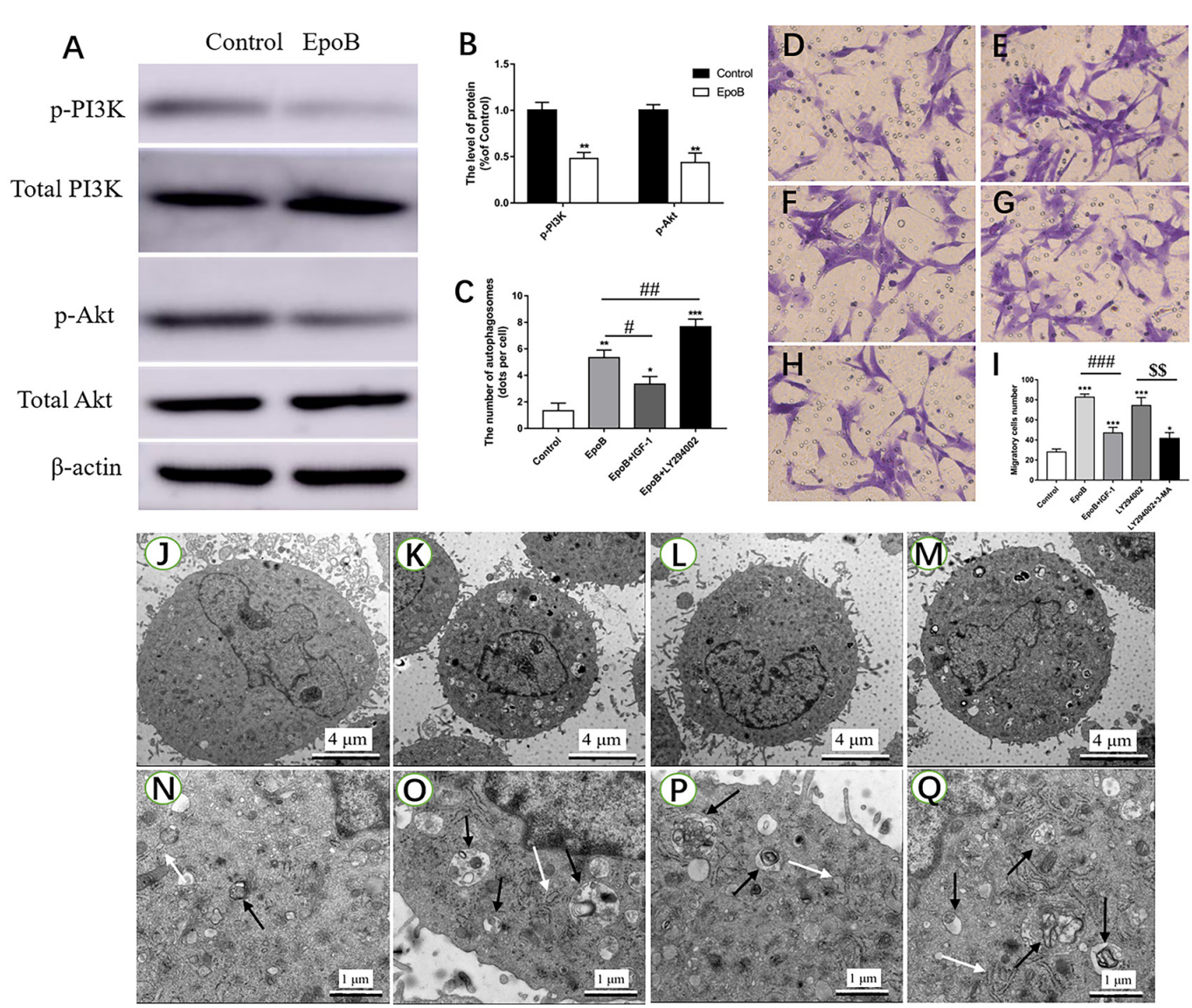

FIGURE 7 | EpoB significantly enhances migration by PI3K/Akt signaling-mediated autophagy in SCs. Representative western blotting (A) and data analysis of p-PI3K and p-Akt in each group (B). (C) Quantification of the total number of autophagosomes in each group. Transwell analysis of migratory cells in the control (D), EpoB (E), EpoB+IGF-1 (F), LY294002 (G), and LY294002+3-MA (H) groups at $24 \mathrm{~h}$ after treatment. (I) Quantification of the total number of migratory cells in each group. TEM analysis showing the formation of autophagosomes in SCs from the control (J,N), EpoB (K,O), EpoB+lGF-1 (L,P), and EpoB+ LY294002 (M,Q) groups at $24 \mathrm{~h}$ after treatment. White arrowheads indicate normal endoplasmic reticula; black arrowheads indicate double-membraned autophagosomes. Scale bars: (J-M)

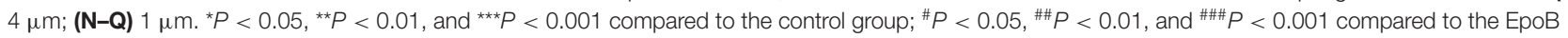
group, ${ }^{\$} P<0.01$ compared to the LY294002 group. Abbreviations: EpoB, epothilone B; 3-MA, 3-methyladenine; SCs, Schwann cells; TEM, transmission electron microscopy.

in different cell types (Zhao et al., 2017). Transforming growth factor- $\beta 1$ (TGF- $\beta 1$ ) promotes cellular invasion and migration by inducing autophagy in breast cancer cells (Huang et al., 2018), while let-7 activates radial migration of newborn neurons via enhancing autophagy (Petri et al., 2017). In contrast, metformin has been found to enhance levels of autophagy and inhibit the migration of endothelial progenitor cells (Li W. D. et al., 2017). In a recent study, stromal cell-derived factor (SDF-1 $\alpha$ or CXCL12) showed a pivotal role in regulating SC migration by enhancing autophagy after facial nerve injury (Gao et al., 2019). We found that EpoB induced autophagy in SCs, and that inhibition of autophagy by 3-MA was capable of inhibiting the EpoB-enhanced SC migration. All of these findings suggest that EpoB might promote SC migration by enhancing autophagy.

The molecules and pathways associated with regulating autophagy in SCs are still unclear. The PI3K/Akt/mTOR signaling pathway plays key roles in controlling cell proliferation, differentiation, growth, survival, and metabolism (Yu and Cui,
2016). PI3K/Akt signaling plays a critical role in maintaining intracellular stability by weakening the expression of neuronal apoptosis-related downstream molecules, such as cleaved caspase-3, Bcl-2 and Bax. This results in Bcl-2/Beclin-1 complex dissociation and subsequently leads to the autophagy response (Gao et al., 2016; Miao et al., 2016). It has been reported that the PI3K kinase inhibitor LY294002 is capable of blocking autophagosome formation ( $\mathrm{Du}$ et al., 2016). In addition, sevoflurane post-conditioning has been found to attenuate traumatic brain injury-induced neuronal apoptosis by promoting autophagy via the PI3K/Akt signaling pathway (He et al., 2018). However, thus far, the role of the PI3K/Akt pathway in regulating autophagy in SCs has been unclear. In the present study, we examined the role of PI3K/Akt signaling in autophagymediated SC migration by EpoB. We found that EpoB treatment results in the inactivation of the PI3K/Akt signaling pathway. Further investigations showed that IGF-1, a PI3K/Akt pathway enhancer, is capable of inhibiting the autophagy and migration 
of SCs induced by EpoB. In contrast, LY294002 (a PI3K/Akt pathway inhibitor) was found to increase cell migration, an effect which was reversed by the autophagy inhibitor 3-MA. Circ-Spidr downregulation has been found to suppress DRG neuron axonal regeneration following injury to the sciatic nerve, partially via modulation of PI3K-Akt signaling (Mao et al., 2019). Maybe EpoB increase axonal regeneration of DRG neurons via the inhibiting of PI3K/Akt pathway immediately, which needs further research. Thus, these findings raise the posibility that $\mathrm{PI} 3 \mathrm{~K} / \mathrm{Akt}$ signaling plays a key role in EpoB-induced autophagy, which is involved in EpoB-enhanced SC migration.

\section{CONCLUSION}

In the present study, it was found that EpoB is capable of promoting axonal regeneration and remyelination, as well as functional recovery in a rat model of nerve crush injury. The beneficial effect of EpoB in the peripheral nervous system was attributable to enhanced SC migration, which was regulated by $\mathrm{PI} 3 \mathrm{~K} /$ Akt signaling-mediated autophagy in SCs. These results underscore the potential therapeutic value of EpoB in enhancing regeneration and functional recovery in cases of PNI.

\section{DATA AVAILABILITY STATEMENT}

All datasets generated for the present study are included in the article.

\section{REFERENCES}

Aszmann, O. C., Korak, K. J., Luegmair, M., and Frey, M. (2008). Bridging critical nerve defects through an acellular homograft seeded with autologous schwann cells obtained from a regeneration neuroma of the proximal stump. J. Reconstr. Microsurg. 24, 151-158. doi: 10.1055/s-2008-1076091

Ballatore, C., Brunden, K. R., Huryn, D. M., Trojanowski, J. Q., Lee, V. M., and Smith, A. B. III. (2012). Microtubule stabilizing agents as potential treatment for Alzheimer's disease and related neurodegenerative tauopathies. J. Med. Chem. 55, 8979-8996. doi: 10.1021/jm301079z

Bar-Yosef, T., Damri, O., and Agam, G. (2019). Dual role of autophagy in diseases of the central nervous system. Front. Cell. Neurosci. 13:196. doi: 10.3389/fncel. 2019.00196

Brogdon, C. F., Lee, F. Y., and Canetta, R. M. (2014). Development of other microtubule-stabilizer families. Anticancer Drugs 25, 599-609. doi: 10.1097/cad.0000000000000071

Campbell, W. W. (2008). Evaluation and management of peripheral nerve injury. Clin. Neurophysiol. 119, 1951-1965. doi: 10.1016/j.clinph.2008.03.018

Cartelli, D., Casagrande, F., Busceti, C. L., Bucci, D., Molinaro, G., Traficante, A., et al. (2013). Microtubule alterations occur early in experimental parkinsonism and the microtubule stabilizer epothilone D is neuroprotective. Sci. Rep. 3:1837. doi: 10.1038/srep01837

Chen, P., Piao, X., and Bonaldo, P. (2015). Role of macrophages in Wallerian degeneration and axonal regeneration after peripheral nerve injury. Acta Neuropathol. 130, 605-618. doi: 10.1007/s00401-015-1482-4

Chen, C., Zhang, J., Sun, L., Zhang, Y., Gan, W. B., Tang, P., et al. (2019). Longterm imaging of dorsal root ganglia in awake behaving mice. Nat. Commun. 10:3087. doi: 10.1038/s41467-019-11158-0

Cheng, H., Huang, H., and Huang, G. (2018). Synthesis and antitumor activity of epothilone B. Eur. J. Med. Chem. 157, 925-934. doi: 10.1016/j.ejmech.2018. 08.055

Du, G., Zhao, Z., Chen, Y., Li, Z., Tian, Y., Liu, Z., et al. (2016). Quercetin attenuates neuronal autophagy and apoptosis in rat traumatic brain injury

\section{ETHICS STATEMENT}

The animal study was reviewed and approved by the Animal Experimentation Ethics Committee of The Fourth Military Medical University, People's Republic of China.

\section{AUTHOR CONTRIBUTIONS}

$\mathrm{JH}, \mathrm{ZL}$, and $\mathrm{HZ}$ conceived and designed the experiments. JZ wrote the manuscript. SL and JG performed the experiments and analyzed the results. $\mathrm{YH}$ and SC isolated SCs from newborn Sprague-Dawley rats and analyzed the purity of the SCs. All authors revised and approved the final version of the manuscript.

\section{FUNDING}

This work was supported by the National Basic Research Program of China (973 Program No. 2014CB542206), the National Natural Science Foundation of China (Grant Nos. 81672148, 81730065, 81601903, and 81802143), and the Excellent Doctoral Thesis of China (Grant No. 201480).

\section{ACKNOWLEDGMENTS}

We thank the technicians Ms. Lifeng Lan, Mrs. Jing Li, and Mr. Xiwang $\mathrm{Hu}$ for their excellent technical assistance.

model via activation of PI3K/Akt signaling pathway. Neurol. Res. 38, 1012-1019. doi: 10.1080/01616412.2016.1240393

Frendo, M. E., da Silva, A., Phan, K. D., Riche, S., and Butler, S. J. (2019). The Cofilin/Limk1 pathway controls the growth rate of both developing and regenerating motor axons. J. Neurosci. 39, 9316-9327. doi: 10.1523/JNEUROSCI.0648-19.2019

Fujimaki, H., Uchida, K., Inoue, G., Matsushita, O., Nemoto, N., Miyagi, M., et al. (2020). Polyglycolic acid-collagen tube combined with collagen-binding basic fibroblast growth factor accelerates gait recovery in a rat sciatic nerve critical-size defect model. J. Biomed. Mater. Res. B Appl. Biomater. 108, 326-332. doi: 10.1002/jbm.b.34391

Gao, Y., Li, J., Wu, L., Zhou, C., Wang, Q., Li, X., et al. (2016). Tetrahydrocurcumin provides neuroprotection in rats after traumatic brain injury: autophagy and the PI3K/AKT pathways as a potential mechanism. J. Surg. Res. 206, 67-76. doi: 10.1016/j.jss.2016.07.014

Gao, D., Tang, T., Zhu, J., Tang, Y., Sun, H., and Li, S. (2019). CXCL12 has therapeutic value in facial nerve injury and promotes Schwann cells autophagy and migration via PI3K-AKT-mTOR signal pathway. Int. J. Biol. Macromol. 124, 460-468. doi: 10.1016/j.ijbiomac.2018.10.212

Gerónimo-Olvera, C., and Massieu, L. (2019). Autophagy as a homeostatic mechanism in response to stress conditions in the central nervous system. Mol. Neurobiol. 56, 6594-6608. doi: 10.1007/s12035-019-1546-X

He, H., Liu, W., Zhou, Y., Liu, Y., Weng, P., Li, Y., et al. (2018). Sevoflurane post-conditioning attenuates traumatic brain injury-induced neuronal apoptosis by promoting autophagy via the PI3K/AKT signaling pathway. Drug Des. Devel. Ther. 12, 629-638. doi: 10.2147/dddt.s158313

Ho, C. J., and Gorski, S. M. (2019). Molecular mechanisms underlying autophagy-mediated treatment resistance in cancer. Cancers 11:E1775. doi: $10.3390 /$ cancers 11111775

Huang, J., Lu, L., Zhang, J., Hu, X., Zhang, Y., Liang, W., et al. (2012). Electrical stimulation to conductive scaffold promotes axonal regeneration and remyelination in a rat model of large nerve defect. PLoS One 7:e39526. doi: 10.1371/journal.pone.0039526 
Huang, F., Shi, Q., Li, Y., Xu, L., Xu, C., Chen, F., et al. (2018). HER2/EGFR-AKT signaling switches TGF $\beta$ from inhibiting cell proliferation to promoting cell migration in breast cancer. Cancer Res. 78, 6073-6085. doi: 10.1158/0008-5472. can-18-0136

Johnson, E. O., Zoubos, A. B., and Soucacos, P. N. (2005). Regeneration and repair of peripheral nerves. Injury 36, S24-S29. doi: 10.1016/j.injury.2005.10.012

Li, X., Huo, C., Xiao, Y., Xu, R., Liu, Y., Jia, X., et al. (2019). Bisdemethoxycurcumin protection of cardiomyocyte mainly depends on Nrf2/HO-1 activation mediated by the PI3K/AKT pathway. Chem. Res. Toxicol. 32, 1871-1879. doi: 10.1021/acs.chemrestox.9b00222

Li, W. D., Li, N. P., Song, D. D., Rong, J. J., Qian, A. M., and Li, X. Q. (2017). Metformin inhibits endothelial progenitor cell migration by decreasing matrix metalloproteinases, MMP-2 and MMP-9, via the AMPK/mTOR/autophagy pathway. Int. J. Mol. Med. 39, 1262-1268. doi: 10.3892/ijmm.2017.2929

Li, Y. L., Sun, J., Hu, X., Pan, Y. N., Yan, W., Li, Q. Y., et al. (2016). Epothilone B induces apoptosis and enhances apoptotic effects of ABT-737 on human cancer cells via PI3K/AKT/mTOR pathway. J. Cancer Res. Clin. Oncol. 142, 2281-2289. doi: 10.1007/s00432-016-2236-y

Li, R., Wu, J., Lin, Z., Nangle, M. R., Li, Y., Cai, P., et al. (2017). Single injection of a novel nerve growth factor coacervate improves structural and functional regeneration after sciatic nerve injury in adult rats. Exp. Neurol. 288, 1-10. doi: 10.1016/j.expneurol.2016.10.015

Liu, B., Xin, W., Tan, J.-R., Zhu, R.-P., Li, T., Wang, D., et al. (2019). Myelin sheath structure and regeneration in peripheral nerve injury repair. Proc. Natl. Acad. Sci. U S A 116, 22347-22352. doi: 10.1073/pnas.1910292116

Mao, S., Huang, T., Chen, Y., Shen, L., Zhou, S., Zhang, S., et al. (2019). CircSpidr enhances axon regeneration after peripheral nerve injury. Cell Death Dis. 10:787. doi: 10.1038/s41419-019-2027-x

Meyer, C., Stenberg, L., Gonzalez-Perez, F., Wrobel, S., Ronchi, G., Udina, E., et al. (2016). Chitosan-film enhanced chitosan nerve guides for long-distance regeneration of peripheral nerves. Biomaterials 76, 33-51. doi: 10.1016/j. biomaterials.2015.10.040

Miao, J., Wang, L., Zhang, X., Zhu, C., Cui, L., Ji, H., et al. (2016). Protective effect of aliskiren in experimental ischemic stroke: up-regulated p-PI3K, p-AKT, Bcl-2 expression, attenuated bax expression. Neurochem. Res. 41, 2300-2310. doi: 10.1007/s11064-016-1944-7

Mizushima, N., Levine, B., Cuervo, A. M., and Klionsky, D. J. (2008). Autophagy fights disease through cellular self-digestion. Nature 451, 1069-1075. doi: $10.1038 /$ nature06639

Motta, C. M. M., Endres, K. J., Wesdemiotis, C., Willits, R. K., and Becker, M. L. (2019). Enhancing Schwann cell migration using concentration gradients of laminin-derived peptides. Biomaterials 218:119335. doi: 10.1016/j.biomaterials. 2019.119335

Novikova, L. N., Pettersson, J., Brohlin, M., Wiberg, M., and Novikov, L. N. (2008). Biodegradable poly- $\beta$-hydroxybutyrate scaffold seeded with Schwann cells to promote spinal cord repair. Biomaterials 29, 1198-1206. doi: 10.1016/j. biomaterials.2007.11.033

Petri, R., Pircs, K., Jönsson, M. E., Akerblom, M., Brattås, P. L., Klussendorf, T., et al. (2017). let-7 regulates radial migration of new-born neurons through positive regulation of autophagy. EMBO J. 36, 1379-1391. doi: 10.15252/embj. 201695235

Rogalska, A., and Marczak, A. (2015). Epothilone B induces human ovarian cancer OV-90 cell apoptosis via external pathway. Environ. Toxicol. Pharmacol. 39, 700-712. doi: 10.1016/j.etap.2015.01.023
Ruschel, J., Hellal, F., Flynn, K. C., Dupraz, S., Elliott, D. A., Tedeschi, A., et al. (2015). Axonal regeneration. Systemic administration of epothilone B promotes axon regeneration after spinal cord injury. Science 348, 347-352. doi: $10.1126 /$ science.aaa2958

Shin, D. H., and Kwon, G. S. (2017). Pre-clinical evaluation of a themosensitive gel containing epothilone B and mTOR/Hsp90 targeted agents in an ovarian tumor model. J. Control Release 268, 176-183. doi: 10.1016/j.jconrel.2017. 10.022

Torigoe, K., Tanaka, H. F., Takahashi, A., Awaya, A., and Hashimoto, K. (1996) Basic behavior of migratory Schwann cells in peripheral nerve regeneration. Exp. Neurol. 137, 301-308. doi: 10.1006/exnr.1996.0030

Wang, L., Liu, Y., Zhou, Y., Wang, J., Tu, L., Sun, Z., et al. (2019). Zoledronic acid inhibits the growth of cancer stem cell derived from cervical cancer cell by attenuating their stemness phenotype and inducing apoptosis and cell cycle arrest through the Erk1/2 and Akt pathways. J. Exp. Clin. Cancer Res. 38:93. doi: 10.1186/s13046-019-1109-Z

Wang, H., Zhang, P., Yu, J., Zhang, F., Dai, W., and Yi, S. (2019). Matrix metalloproteinase 7 promoted Schwann cell migration and myelination after rat sciatic nerve injury. Mol. Brain 12:101. doi: 10.1186/s13041-019-0516-6

Xia, B., Huang, L., Zhu, L., Liu, Z., Ma, T., Zhu, S., et al. (2016). Manipulation of Schwann cell migration across the astrocyte boundary by polysialyltransferase-loaded superparamagnetic nanoparticles under magnetic field. Int. J. Nanomedicine 11, 6727-6741. doi: 10.2147/ijn. s122358

Yang, Y., Zhang, X., Ge, H., Liu, W., Sun, E., Ma, Y., et al. (2018). Epothilone $\mathrm{B}$ benefits nigrostriatal pathway recovery by promoting microtubule stabilization after intracerebral hemorrhage. J. Am. Heart Assoc. 7:e007626. doi: 10.1161/jaha.117.007626

Yu, J. S., and Cui, W. (2016). Proliferation, survival and metabolism: the role of $\mathrm{PI} 3 \mathrm{~K} / \mathrm{AKT} / \mathrm{mTOR}$ signalling in pluripotency and cell fate determination. Development 143, 3050-3060. doi: 10.1242/dev.137075

Yu, B., Zhou, S., Yi, S., and Gu, X. (2015). The regulatory roles of non-coding RNAs in nerve injury and regeneration. Prog. Neurobiol. 134, 122-139. doi: 10.1016/j. pneurobio.2015.09.006

Zhang, L., Ma, Z., Smith, G. M., Wen, X., Pressman, Y., Wood, P. M., et al. (2009). GDNF-enhanced axonal regeneration and myelination following spinal cord injury is mediated by primary effects on neurons. Glia 57, 1178-1191. doi: 10.1002/glia.20840

Zhao, Z., Li, X., and Li, Q. (2017). Curcumin accelerates the repair of sciatic nerve injury in rats through reducing Schwann cells apoptosis and promoting myelinization. Biomed. Pharmacother. 92, 1103-1110. doi: 10.1016/j.biopha. 2017.05.099

Conflict of Interest: The authors declare that the research was conducted in the absence of any commercial or financial relationships that could be construed as a potential conflict of interest.

Copyright (C) 2020 Zhou, Li, Gao, Hu, Chen, Luo, Zhang, Luo and Huang. This is an open-access article distributed under the terms of the Creative Commons Attribution License (CC BY). The use, distribution or reproduction in other forums is permitted, provided the original author(s) and the copyright owner(s) are credited and that the original publication in this journal is cited, in accordance with accepted academic practice. No use, distribution or reproduction is permitted which does not comply with these terms. 